American Journal of Infectious Diseases 5 (1): 26-30, 2009

ISSN 1553-6203

(C) 2009 Science Publications

\title{
Cocaine Exposure Results in Formation of Dendritic Varicosity in Rat Primary Hippocampal Neurons
}

\author{
${ }^{1}$ Honghong Yao, ${ }^{1}$ Crystal Bethel-Brown, ${ }^{2}$ Anil Kumar and ${ }^{1}$ Shilpa Buch \\ ${ }^{1}$ Department of Molecular and Integrative Physiology, University of Kansas Medical Center, \\ Kansas City, KS 66160 \\ ${ }^{2}$ Department of Pharmacology, School of Pharmacy, University of Missouri-Kansas City, \\ Kansas City, MO 64108
}

\begin{abstract}
Problem statement: It has been well documented that drugs of abuse such as cocaine can cause enhanced progression of HIV-Associated Neuropathological Disorders (HAND), the underlying mechanisms mediating these effects remain poorly understood. Approach: In present study, we explored the impact of cocaine exposure (I and $10 \mu \mathrm{M}$ ) on the dendritic beading in rat primary hippocampal neurons. Using the approach of transfection with green fluorescent protein, we observed significant dendritic swelling in hippocampal neurons exposed to $10 \mu \mathrm{M}$ but not $1 \mu \mathrm{M}$ of cocaine when compared with the saline treated group. Results: Cocaine exposure also resulted in decreased expression of the synaptic plasticity gene, Arc as evidenced by Western blotting. Intriguingly, cocaine exposure of primary neurons in the presence of the neurotoxin-HIV envelope protein gp 120, resulted in increased enhancement of neuronal beading as compared with exposure of neurons to either agent alone. Conclusion: Taken together these findings imply that cocaine in co-operation with HIV protein exacerbates neuronal damage in the brains of HIV-infected cocaine abusers.
\end{abstract}

Key words: Cocaine, HIV-1-associated neurological disorders, hippocampal neurons, dendritic swelling

\section{INTRODUCTION}

Mounting evidence suggests that drugs of abuse accelerate the incidence and progression of HIV-1Associated Neurological Disorders (HAND) ${ }^{[1-3]}$. Drugabusing HIV-1 positive individuals exhibit more severe cognitive impairment compared with non drug-abusing HIV positive counterparts. Epidemiological studies on abused drug users and AIDS link abuse of cocaine (by different routes), even more than other drugs, to increased incidence of HIV prevalence and progression to AIDS $^{[4-6]}$. Cocaine targets the CNS and is associated with brain dysfunction. Despite the recognized impact of the abuse of psychostimulants on the clinical course of HIV-1-associated brain pathology ${ }^{[1]}$, mechanisms underlying the ability of these drugs to enhance the pathological effects of HIV-1 in the brain remain elusive.

Neurologic impairment in patients with HAND correlates well with injury to dendrites and synapses ${ }^{[7]}$. This clinical phenomenon has been shown in Alzheimer disease $^{[8]}$ and dendritic injury in both the diseases is characterized by focal swelling or beading, loss of spines, and reductions in overall dendritic and synaptic areas $^{[9,10]}$. Therefore, synaptic protection represents an area of considerable therapeutic interest. Previous study indicated that In vitro exposure of neurons to glutamate resulted in dendritic beading ${ }^{[11]}$ and loss of synaptic transmission. Mechanism by which cocaine causes dendritic injury remains an enigma. A better understanding of how HIV-1 and drugs of abuse affect dendrites may lead to development of more effective therapeutic interventions for the interlinked epidemic of HIV and drug abuse. The current study is aimed at exploring the effect of cocaine and/or HIV proteins on neuronal health with emphasis on dendritic morphology.

\section{MATERIALS AND METHODS}

Primary neuronal cultures: Primary neurons were prepared as described earlier with slight modifications. Briefly, hippocampus tissues were dissected from embryonic day 13-14 Sprague-Dawley rats and dissociated with a mild mechanical trituration.

Corresponding Author: J. Shilpa Buch, Department of Molecular and Integrative Physiology, 5000 Wahl Hall East, University of Kansas Medical Center, 3901 Rainbow Blvd, Kansas City, KS 66160

Tel: (913) 5887389 Fax: (913) 5885599 
Dissociated cells were seeded first at $5 \times 10^{5}$ cells well $^{-1}$ on 24-well culture plates and maintained in Dulbecco's modified Eagle's medium/F12 supplemented with 10\% fetal bovine serum and $1 \%$ antibiotic. One day later the cultures were supplemented with serum-free neurobasal medium containing B27 (50:1), $2 \mathrm{mM}$ glutamax, and $1 \%$ antibiotic. After 7 days, the initial plating cultures consisted of $90 \%$ neuron-specific nuclear-proteinimmunereactive neurons.

Green fluorescent protein transfection: Neurons were transfected at 2-3 d in culture with the Green Fluorescent Protein (GFP) plasmid using the DOSPER Liposomal Transfection Reagent (Boehringer Mannheim, Indianapolis, IN) as described the previous study ${ }^{[7]}$. These conditions were selected to yield a transfection efficiency of $0.01 \%$, permitting the study of individual neurons. Neuronal cell bodies expressed GFP the day after transfection with development of neuritis over subsequent days. GFP fluorescence was stable in a number of neurons for at least 3 weeks and revealed the neuronal arbor, including axons, dendrites, and dendritic spines.

Western blotting

Treated cells were lysed using the Mammalian Cell Lyses kit (Sigma, St. Louis, MO). Western blots were then probed with antibodies recognizing the Arc antibody (Santa Cruz). The secondary antibodies were alkaline phosphates conjugated to goat anti mouse/rabbit IgG (1:5000). Signals were detected by chemiluminescence's (Pierce, Rockford, IL). Western blot experiments are repeated three times individually, representative examples are presented in the figures.

Immunocytochemistry: For MAP-2 staining, neurons were fixed for $30 \mathrm{~min}$ with $4 \%$ paraformaldehyde at room temperature. The fixed cells were permeabilized with $1 \%$ Triton X100 for 30 min, followed by staining with MAP-2 antibody. Briefly, after blocking with 3\% normal goat serum, $0.1 \%$ Triton X-100 and 1\% BSA, sections were incubated with rabbit anti-MAP-2 (1:1000, Chemicon, Temecula, CA), washed in PBS $(0.1 \mathrm{M})$ and incubated with secondary antibody.

Statistical analysis: Data were expressed as mean \pm SD. Significance of differences between control and cocaine-treated samples was determined by one-way ANOVA followed by the post hoc Least-SignificantDifference (LSD) test. Values of $\mathrm{p}<0.05$ were considered to be statistically significant.

\section{RESULTS}

Cocaine caused dendritic varicosity in primary hippocampus neurons: To examine effects of cocaine exposure on dendrites, primary neurons were treated with different concentrations of cocaine followed by measuring dendritic varicosity. As shown in Fig. 1, $10 \mu \mathrm{M}$ of cocaine caused dendritic varicosity throughout the dendritic arbors within $120 \mathrm{~min}$ of treatment, an effect that was not observed with $1 \mu \mathrm{M}$ cocaine. 2. Cocaine regulated Arc expression in hippocampal neurons

Since neuronal plasticity is exemplified by the expression of the Arc gene, we next sought to explore the expression of Arc Western blot analysis in parallel with the dendritic varicosity. Interestingly, exposure of neurons to $10 \mu \mathrm{M}$ of cocaine, that resulted in enhanced dendritic varicosity, correspondingly demonstrated decreased Arc expression (Fig. 2).

Rat primary neurons exposed to different concentrations of cocaine were monitored for cocainemediated Arc expression. Exposure of neurons to 10 $\mu \mathrm{M}$ of cocaine resulted in decreased Arc expression while exposure of cell to $1 \mu \mathrm{M}$ cocaine resulted in increased Arc expression. The figure is a representative image of three independent experiments

Gp120 and cocaine caused enhanced dendritic varicosity formation: Drugs of abuse are known to accelerate the progress of $\mathrm{HIV}^{[1,12]}$. We next sought to explore the effect of both cocaine and the HIV protein gp120 on dendritic varicosity. Rat primary neurons cotreated with gp120 and cocaine were examined for somatodendritic microtubule-associated protein MAP2 using staining by immunofluoresence. Control group revealed neurons with round somata and an intricately branched dendritic arbor with smooth dendrites.
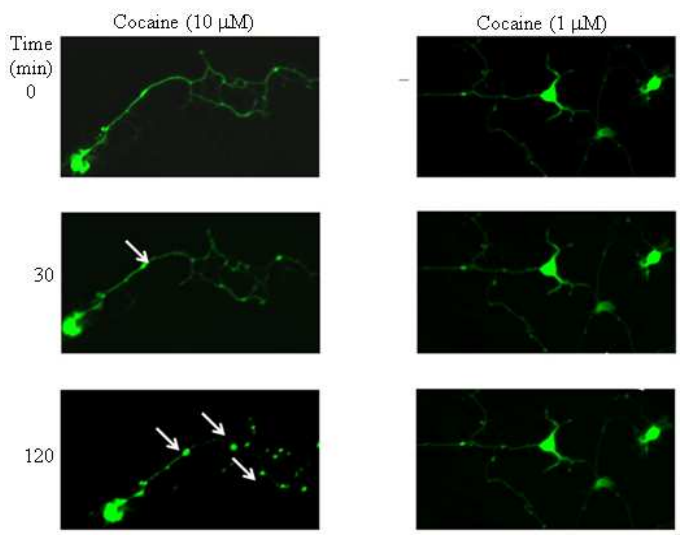

Fig. 1: Cocaine exposure resulted in formation of dendritic varicosity in primary hippocampus neurons. Rat hippocampal neurons exposed to $10 \mu \mathrm{M}$ but not $1 \mu \mathrm{M}$ cocaine developed dendritic varicosities. The image shown is a representative picture of three independent experiments. Images were captured on a timelapse confocal microscope. Arrows indicate the sites of dendritic varicosity formation 


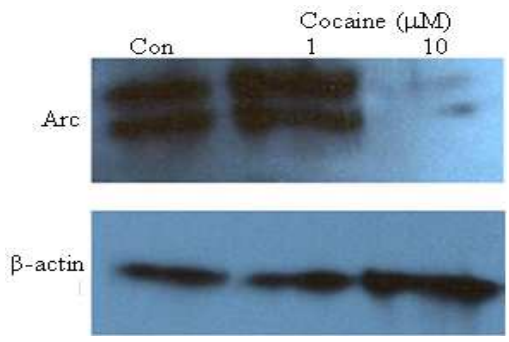

Fig. 2: Cocaine regulated Arc expression in hippocampal neurons

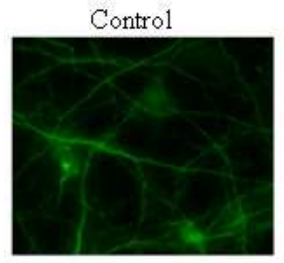

gp 120

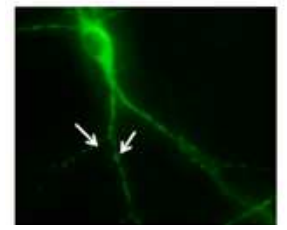

(a)

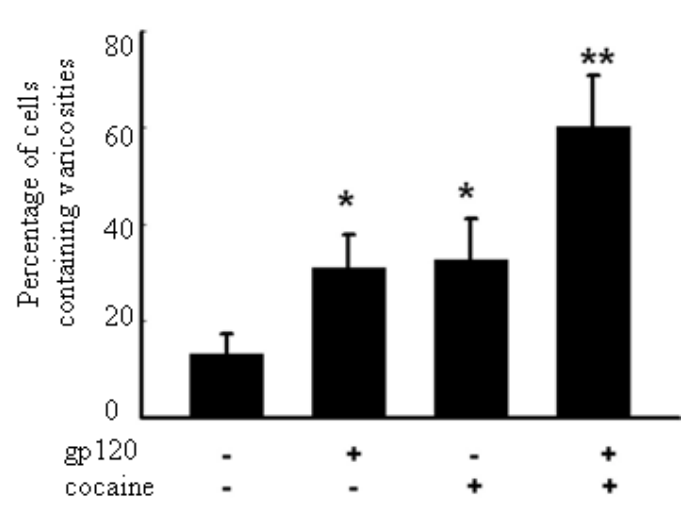

(b)

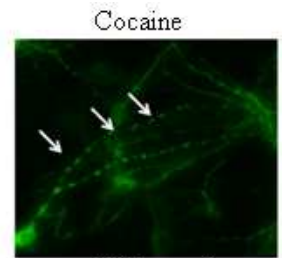

gp $120+$ cocaine

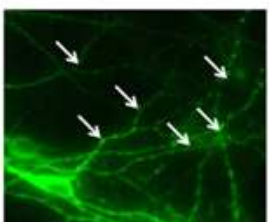

Fig. 3: Gp120 and cocaine caused enhanced dendritic varicosity formation. (a) MAP2 immunofluorescence of primary hippocampal neurons exposed to cocaine, gp120 and cocaine plus gp120 demonstrated increased dendritic varicosity formation in the presence of both cocaine and gp120 as compared with neurons exposed to either agent alone. Arrows indicate the sites of dendritic varicosity formation. (b) Quantification of percentage of cells with dendritic varicosities. All the data are presented as mean $\pm \mathrm{SD}$ of four individual experiments. $* \mathrm{p}<0.05 ; * * \mathrm{p}<0.01$ vs control group
Exposure of neurons to either cocaine $(10 \mu \mathrm{M})$ or gp120 (200 $\mathrm{ng} \mathrm{mL} \mathrm{mL}^{-1}$ ) alone for $120 \mathrm{~min}$ demonstrated the appearance of dendritic varicosity along the length of the dendrite. This effect was further enhanced in neurons exposed to both cocaine as well as gp120, with increased dendritic varicosity formation as shown in Fig. 3.

\section{DISCUSSION}

While there is ample evidence suggesting that drugs of abuse such as cocaine can co-operate with HIV-1 to induce neurotoxicity ${ }^{[3]}$, the mechanisms involved in this process remain elusive. The present study demonstrated that cocaine exposure of hippocampal neurons resulted in increased neuronal beading. Further findings determined that the mechanisms underlying the cocaine exposure-mediated impairment of neuronal dendrites in primary hippocampal neurons involved down-regulation of the expression of the neuronal plasticity gene Arc. Additionally, exposure of neurons to HIV-1 envelope protein gp120 resulted in enhanced loss of dendrites of neurons exposed to cocaine.

Acute dendritic swelling and spine loss are pathological hallmarks of brain injury and spine loss is associated with focal dendritic swelling ${ }^{[13]}$. In present study, we demonstrated that cocaine exposure damaged neuronal dendrites in cell culture, a finding that was consistent with the previous study demonstrating that in locus coeruleus neurons, cocaine treatment decreased both the number of cell initiating neuritis as well as the length of neuritis ${ }^{[14]}$. Although there are conflicting reports indicating that acute cocaine exposure increased spine density and synaptic plasticity of the dopaminergic neurons in the ventral tegmental area in vivo ${ }^{[15]}$, the cellular mechanism(s) underlying the effects of cocaine on neuronal plasticity remain unclear. In our study, it was interesting to note that the spine loss occurred over short intervals following treatment with $10 \mu \mathrm{M}$ of cocaine, but not with $1 \mu \mathrm{M}$ of cocaine. These findings likely suggest dose-dependent differential effects of cocaine on neuronal plasticity. Long-lasting changes in synaptic efficacy are accompanied by the expression of immediate early genes, including the activity-regulated cytoskeletonassociated gene Arc, also known as Arg3.1 ${ }^{[16]}$. In the present study, we observed that different concentrations of cocaine exerted distinct effects on Arc expression, with1 $\mu \mathrm{M}$ of cocaine increasing Arc expression, while $10 \mu \mathrm{M}$ of cocaine exhibiting the opposite effects. Based upon the effects of cocaine on the neuronal dendrites, down-regulation of Arc expression may contribute to 
the damage of neuronal dendrites. Up-regulation of Arc expression following exposure of cells to $1 \mathrm{uM}$ cocaine (this concentration was identical to the physiological concentration) ${ }^{[17]}$ was consistent with the previous reports demonstrating cocaine-mediated up-regulation of Arc expression primarily in striatum, prefrontal cortex and the hippocampus ${ }^{[18]}$.

Neurologic impairment in patients with HAND correlates well with injury to dendrites and synapses ${ }^{[7]}$. Despite significant progress in understanding the molecular mechanisms underlying HIV-1 infection and drug abuse-induced neurotoxicity, very few studies have directly examined the impact of HIV-1 infection on dendrites and whether such alterations may contribute to HIV-1-associated neurologic dysfunction.

\section{CONCLUSION}

Present study demonstrated enhanced neuronal beading in the presence of cocaine and HIV proteins as compared with neurons exposed to either agent alone thus shedding light on the exacerbated toxicity mediated by drugs of abuse such as cocaine in the presence of HIV-infection. A better understanding of how HIV-1 and drugs of abuse together can affect the molecular and cellular mechanisms of dendrite damage, can lead to development of new therapeutic approaches targeting preservation of dendrites.

\section{ACKNOWLEDGEMENT}

This study was supported by grants RR016443, MH-068212, DA020392, DA023397 and DA024442 from the National Institutes of Health (SB).

\section{REFERENCES}

1. Aksenov, M.Y. et al., 2006. Cocaine-mediated enhancement of Tat toxicity in rat hippocampal cell cultures: The role of oxidative stress and D1 dopamine receptor. Neurotoxicology, 27: 217-228. http://cat.inist.fr/?aModele $=$ afficheN\&cpsidt $=17583603$

2. Gurwell, J.A. et al., 2001. Synergistic neurotoxicity of opioids and human immunodeficiency virus-1 Tat protein in striatal neurons in vitro. Neuroscience, 102: 555-563. http://www.ncbi.nlm.nih.gov/pubmed/11226693

3. Turchan, J. et al., 2001. Estrogen protects against the synergistic toxicity by HIV proteins, methamphetamine and cocaine. BMC Neurosci., 2: 3. DOI: $10.1186 / 1471-2202-2-3$
4. Anthony, J.C., et al., 1991. New evidence on intravenous cocaine use and the risk of infection with human immunodeficiency virus type 1 . Am. J. Epidemiol., 134: 1175-1189. http://aje.oxfordjournals.org/cgi/content/abstract/13 4/10/1175

5. Baldwin, G.C., M.D. Roth, and D.P. Tashkin, 1998. Acute and chronic effects of cocaine on the immune system and the possible link to AIDS. J. Neuroimmunol., $\quad$ 83: 133-138. http://www.ncbi.nlm.nih.gov/pubmed/9610681

6. Doherty, M.C. et al., 2000. Correlates of HIV infection among young adult short-term injection drug users. AIDS, 14: 717-726. http://www.ncbi.nlm.nih.gov/pubmed/10807195

7. Bellizzi, M.J. et al., 2005. Synaptic activity becomes excitotoxic in neurons exposed to elevated levels of platelet-activating factor. J. Clin. Invest., 115: 3185-3192. DOI: 10.1172/JCI25444

8. Dekosky, S.T. and S.W. Scheff, 1990. Synapse loss in frontal cortex biopsies in Alzheimer's disease: correlation with cognitive severity. Ann. Neurol., 27: 457-464. http://www.ncbi.nlm.nih.gov/pubmed/2360787

9. Masliah, E. et al., 1992. Selective neuronal vulnerability in HIV encephalitis. J. Neuropathol. Exp. Neurol., 51: 585-593. http://www.ncbi.nlm.nih.gov/pubmed/1484289

10. Masliah, E. et al., 1994. Synaptic and neuritic alterations during the progression of alzheimer's disease. Neurosci. Lett., 174: 67-72. http://www.ncbi.nlm.nih.gov/pubmed/7970158

11. Park, J.S., M.C. Bateman and M.P. Goldberg, 1996. Rapid alterations in dendrite morphology during sublethal hypoxia or glutamate receptor activation. Neurobiol. Dis., 3: 215-227. DOI: 10.1006/nbdi.1996.0022

12. Goodkin, K. et al., 1998. Cocaine abuse and HIV-1 infection: Epidemiology and neuropathogenesis. J. Neuroimmunol., 83: 88-101. http://www.ncbi.nlm.nih.gov/pubmed/9610677

13. Hasbani, M.J. et al., 2001. Dendritic spines lost during glutamate receptor activation reemerge at original sites of synaptic contact. J. Neurosci., 21: 2393-2403.

http://www.jneurosci.org/cgi/content/abstract/21/7/2393

14. Dey, S., et al., 2006. Specificity of prenatal cocaine on inhibition of locus coeruleus neurite outgrowth. Neuroscience, 139: 899-907. DOI: 10.1016/j.neuroscience.2005.12.053 
15. Sarti, F. et al., 2007. Acute cocaine exposure alters spine density and long-term potentiation in the ventral tegmental area. Eur. J. Neurosci., 26: 749-756. http://cat.inist.fr/?aModele $=$ afficheN\&cpsidt $=18970270$

16. Rodriguez, J.J., et al., 2005. Long-term potentiation in the rat dentate gyrus is associated with enhanced Arc/Arg3.1 protein expression in spines, dendrites and glia. Eur. J. Neurosci., 21: 2384-2396.

http://www.ncbi.nlm.nih.gov/pubmed/15932597

17. Dey, S. and D.M. Snow, 2007. Cocaine exposure in vitro induces apoptosis in fetal locus coeruleus neurons through TNF-alpha-mediated induction of Bax and phosphorylated c-Jun NH (2)-terminal kinase. J. Neurochem., 103: 542-556. DOI: 10.1111/j.1471-4159.2007.04750.x
18. Fumagalli, F. et al., 2006. Corticostriatal upregulation of activity-regulated cytoskeletalassociated protein expression after repeated exposure to cocaine. Mol. Pharmacol., 70: 1726-1734. DOI: $10.1124 / \mathrm{mol} .106 .026302$ 